\title{
CLUSTERED SPARSITY AND SEPARATION OF CARTOON AND TEXTURE
}

\author{
GITTA KUTYNIOK
}

\begin{abstract}
Natural images are typically a composition of cartoon and texture structures. A medical image might, for instance, show a mixture of gray matter and the skull cap. One common task is to separate such an image into two single images, one containing the cartoon part and the other containing the texture part. Recently, a powerful class of algorithms using sparse approximation and $\ell_{1}$ minimization has been introduced to resolve this problem, and numerous inspiring empirical results have already been obtained.

In this paper we provide the first thorough theoretical study of the separation of a combination of cartoon and texture structures in a model situation using this class of algorithms. The methodology we consider expands the image in a combined dictionary consisting of a curvelet tight frame and a Gabor tight frame and minimizes the $\ell_{1}$ norm on the analysis side. Sparse approximation properties then force the cartoon components into the curvelet coefficients and the texture components into the Gabor coefficients, thereby separating the image. Utilizing the fact that the coefficients are clustered geometrically, we prove that at sufficiently fine scales arbitrarily precise separation is possible. Main ingredients of our analysis are the novel notion of cluster coherence and clustered/geometric sparsity. Our analysis also provides a deep understanding on when separation is still possible.
\end{abstract}

\section{INTRODUCTION}

Natural images are typically a composition of 'cartoon' and 'texture'. Think, for instance, of a medical image in which we might see a mixture of gray matter and the skull cap, or, more general, a mixture of tissue and bones. Often, it is essential to very cleanly separate the cartoon from the texture part, i.e., to generate two single images from the original one, for separate analysis. However, each educated person these days would say that it is entirely impossible to solve a problem with only one known datum and two unknowns. Intriguingly, the deep reason for why this separation is possible in the considered situation is the stark morphological difference between both structures.

The main idea of the empirical results exploiting applied harmonic analysis methodologies (see, e.g., [49, 50, 51, 52, 25] - in comparison to PDE-based separation methods (see exemplary [56] and references therein), use the fact that there exist sparsifying dictionaries for

Key words and phrases. Curvelets. Gabor Frames. $\ell_{1}$-Minimization. Parabolic Scaling. Shearlets. Sparsity.

The author would like to thank David Donoho for various discussions on this and related topics. She is grateful to the Department of Statistics at Stanford University and the Department of Mathematics at Yale University for their hospitality and support during her visits. The author acknowledges support by the Einstein Foundation Berlin, by Deutsche Forschungsgemeinschaft (DFG) Heisenberg fellowship KU 1446/8, Grant SPP-1324 KU 1446/13 and DFG Grant KU 1446/14, and by the DFG Research Center MATHEON "Mathematics for key technologies" in Berlin. 
cartoons and for textures. Dictionary learning on natural images indicates that a curvelet (or shearlet) system might be best adapted to the cartoon part, whereas a Gabor system might be best adapted to the texture part (cf. [46]). Roughly speaking, the image is then expanded into a combined dictionary of curvelets and a Gabor system, and the $\ell_{1}$ norm on the analysis side is minimized. Sparse approximation properties then force the cartoon components into the curvelet coefficients and the texture components into the Gabor coefficients, thereby separating the image. However, no theoretical results are available to date which provide a deep mathematical understanding of why separation is possible.

In the paper [19] (see also [38]), the apparently similar problem of separating pointand curve-like structures was considered, a distributional model was developed, and clustered/geometric sparsity and cluster coherence were introduced to derive an asymptotic separation result; asymptotic in the scale. However, the analysis required for the situation of cartoon and texture differs significantly, since a Gabor system does not exhibit a scaling component, and a distributional model is not feasible for the texture part. Our analysis will though still be based on the novel viewpoint of clustered/geometric sparsity and cluster coherence.

There exists an intriguing connection with quasicrystals [28], since those objects can be regarded as both periodic (texture-like) as well as non-periodic with sharp boundaries (cartoonlike). This raises the question of how periodization of a cartoon can make it separable from a single cartoon. This highly fascinating question is though beyond the scope of this paper, but one goal for future exploration.

1.1. Model of Cartoon Part. Intuitively, cartoons are smooth image parts separated from other areas by an edge. The first model of cartoons has been introduced in [5], and this is what we intend to use also here. The basic idea is to choose a closed boundary curve and then fill the interior and exterior part with $C^{2}$ functions. Here, we will adapt the definition from [5] slightly.

We define a model $\mathcal{C}$ of a cartoon as follows: Let $\tau$ be a closed, non-intersecting, and regular curve in $\mathbb{R}^{2}$, i.e., $\tau$ is $C^{\infty}$ and of finite arclength, and let $B_{\tau}$ denote the interior of $\tau$. Then we choose $\mathcal{C}$ as

$$
\mathcal{C}=f_{0}+f_{1} \cdot 1_{B_{\tau}}
$$

where $f_{0}, f_{1} \in L^{2}\left(\mathbb{R}^{2}\right) \cap C^{2}\left(\mathbb{R}^{2}\right)$ with compact support. An example of such a cartoon is illustrated in Figure 1.

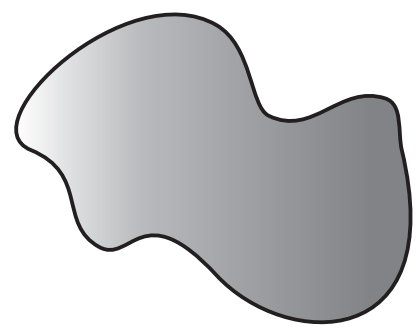

FiguRE 1. Example of a cartoon-like image. 
To set up the analysis in a reasonable way, we will need to restrict our model of cartoons slightly, which will be done in Subsection 1.5.

1.2. Model of Texture Part. People have debated for years over an appropriate model for the texture content of an image. The outcome can be seen nowadays in an extensive variety of texture models both for the deterministic as well as statistical setting. We would like to remind the reader of models such as Gaussian random fields, or the $H^{1}$-model [47]. All of those emphasize different features of texture content such as for the $H^{-1}$-model a special oscillatory behavior 'dual' to cartoons, which are sometimes modeled as $B V$-functions, is highlighted. However, certainly even an intersection of all these models is far from being capable of accurately describing texture, especially since the perception of texture also differs from observer to observer. In the area of computer vision very interesting studies have been performed, for instance by Zucker [2, 22, 23], which we would like to bring to the reader's attention.

Our understanding of texture is a function which is concentrated on a small part of the image with a periodic structure. This justifies the use of a very 'classical' approach by modeling texture as a function which is sparse in a Gabor frame. However, we would like the reader to be aware of the fact that certainly also this deterministic model is quite simplified and possible extensions could be incorporated in it, which we would like to briefly discuss:

- Warping: Let us consider an image containing a table cloth such as the famous 'Barbara' image. Remembering that this table cloth was in fact folded, we could think of improving our model by using diffeomorphism to model this warping effect, which was done in [10].

- Affine transform: Again abusing 'Barbara' as an example, we recall that the table cloth was in addition also sheared. This could be handled by application of an affine transform to the texture content.

- Windowing: Our running example also reminds us that texture - here a pattern of a table cloth - could be contained in a polygon, e.g., in particular, the pattern is broken. Thus refining our model further would force us to consider windowed 'Gabor' texture models, which, in particular, requires a careful treatment of the boundaries. This also illustrates how delicate the distinction of texture from cartoons can be, and let us wonder where in such a transition to draw the line between texture and cartoons.

- Textons: Finally, textures commonly do not appear as singletons, but might also overlap. This can be attacked by considering a model of texture which is a linear combination of shifted 'Gabor' texture model with coefficients being iid to decouple the texture parts. As a reference for this general concept which in literature is normally referred to as textons, we would like to mention [57].

We now define our model for texture as follows: Let $g \in L^{2}\left(\mathbb{R}^{2}\right)$ be a window with $|g(x)| \sim e^{-|x|}, \hat{g} \in C^{\infty}\left(\mathbb{R}^{2}\right)$, and frequency support supp $\hat{g} \subseteq[-1,1]^{2}$ satisfying

$$
\sum_{n \in \mathbb{Z}}|\hat{g}(\xi+n)|^{2}=1, \quad \xi \in \mathbb{R}^{2} .
$$

For a 'size parameter' $s>0$, let $g_{s}$ denote the $L^{2}$-normalized scaled version of $g$ defined by

$$
g_{s}(x)=s \cdot g(s x) \text {. }
$$


With this choice, $\hat{g}_{s}(\xi)=s^{-1} \cdot \hat{g}\left(s^{-1} \xi\right)$, and hence

$$
\operatorname{supp} \hat{g}_{s} \subseteq[-s, s]^{2}
$$

as well as

$$
\sum_{n \in \mathbb{Z}}\left|\hat{g}_{s}(\xi+s n)\right|^{2}=s^{-2}, \quad \xi \in \mathbb{R}^{2} .
$$

Further, let $\left(d_{m, n}\right)_{m, n \in \mathbb{Z}^{2}}$ be a sequence of complex numbers belonging to $\ell_{1} \cap \ell_{2}$. Then the function of interest to us as a model for texture is defined by

$$
\mathcal{T}_{s}=\sum_{m, n \in \mathbb{Z}^{2}} d_{m, n} g_{s}\left(x-\frac{m}{2 s}\right) e^{i(s n)^{\prime} x} .
$$

1.3. Model of Image Composed of a Cartoon- and Texture-Part. Having introduced both constituents, we now assume that the image

$$
f_{s}=\mathcal{C}+\mathcal{T}_{s}
$$

is observed, however both the cartoon component $\mathcal{C}$ as well as the texture component $\mathcal{T}_{s}$ are unknown to us. Thus we face the task of extracting those from $f_{s}$, which is what we will analyze in this paper.

For the purpose of matching the energy of cartoon- and texture-part so that the separation becomes non-trivial, in Subsection 1.5, we will link $s$ to a particular other parameter.

1.4. Sparsifying Overcomplete Systems. Since we intend to utilize $\ell_{1}$ minimization to separate $\mathcal{C}$ and $\mathcal{T}_{s}$ in the sense of extracting $\mathcal{C}$ and $\mathcal{T}_{s}$ from $f_{s}$, appropriate systems need to be selected which provide (relatively) sparse representations for those components. Of interest to us are the following two systems:

- Curvelets - a highly directional tight frame with increasingly anisotropic elements at fine scales.

- Gabor tight frame - a tight frame with time-frequency-balanced elements.

These systems are constructed as follows. Using the function $g$ introduced in Subsection 1.2, we first define the Gabor tight frame at spatial position index $m$ and frequency position index $n$ by the Fourier transform

$$
\left(\hat{g}_{s}\right)_{\lambda}(\xi)=\hat{g}_{s}(\xi-s n) e^{i \frac{m}{2 s}{ }^{\prime} \xi},
$$

where we let $\lambda=(m, n)$ index spatial and frequency position. The bandsize is indexed by the parameter $s$. It follows from [12] that this system constitutes a tight frame for $L^{2}\left(\mathbb{R}^{2}\right)$.

To define curvelets, we let $W$ be the inverse Fourier transform of a wavelet, where $W$ belongs to $C^{\infty}(\mathbb{R})$ and is compactly supported on $[1 / 2,2]$, for instance, suitably scaled LemarièMeyer wavelets possess these properties. Further, we choose a "bump function" $V \in C^{\infty}$, which is compactly supported on $[-1,1]$. We then define continuous curvelets at scale $a>0$, orientation $\theta \in[0, \pi)$, and spatial position $b \in \mathbb{R}^{2}$ by their Fourier transforms

$$
\hat{\gamma}_{a, b, \theta}(\xi)=a^{\frac{3}{4}} \cdot W(a|\xi|) V\left(a^{-1 / 2}(\omega-\theta)\right) \cdot e^{i b^{\prime} \xi} .
$$


See $[5,6]$ for more details. The curvelet tight frame is then (essentially) defined as a sampling of $b$ on a series of regular lattices

$$
\left\{R_{\theta_{j, \ell}} D_{a_{j}} \mathbb{Z}^{2}\right\}, \quad j \geq j_{0}, \quad \ell=0, \ldots, 2^{\lfloor j / 2\rfloor}-1,
$$

where $R_{\theta}$ is planar rotation by $\theta$ radians, $a_{j}=2^{-j}, \theta_{j, \ell}=\pi \ell / 2^{j / 2}, \ell=0, \ldots, 2^{j / 2}-1$, and $D_{a}$ is anisotropic dilation by $\operatorname{diag}(a, \sqrt{a})$, i.e., the curvelets at scale $j$, orientation $\ell$, and spatial position $k=\left(k_{1}, k_{2}\right)$ are given by the Fourier transform

$$
\hat{\gamma}_{\eta}(\xi)=2^{-j \frac{3}{4}} \cdot W\left(|\xi| / 2^{j}\right) V\left(\left(\omega-\theta_{j, \ell}\right) 2^{j / 2}\right) \cdot e^{i\left(R_{\theta_{j, \ell}} D_{2-j} k\right)^{\prime} \xi}
$$

where $\eta=(j, k, \ell)$ index scale, orientation, and scale. (For a precise statement, see [7, Section 4.3, pp. 210-211]).

Using the same window $W$, we can construct a family of filters $F_{j}$ with transfer functions

$$
\hat{F}_{j}(\xi)=W\left(|\xi| / 2^{j}\right), \quad \xi \in \mathbb{R}^{2} .
$$

These filters allow us to decompose a function $f$ into pieces $f_{j}$ with different scales, the piece $f_{j}$ at subband $j$ arises from filtering $f$ using $F_{j}$ :

$$
f_{j}=F_{j} \star f ;
$$

the Fourier transform $\hat{f}_{j}$ is supported in the annulus with inner radius $2^{j-1}$ and outer radius $2^{j+1}$. Because of our assumption on $W$, we can reconstruct the original function from these pieces using the formula

$$
f=\sum_{j} F_{j} \star f_{j}, \quad f \in L^{2}\left(\mathbb{R}^{2}\right) .
$$

This allows us to split the seeked decomposition of the observed image $f_{s}$ (see (1)) into infinitely many decompositions depending on scale $j$ by setting

$$
\mathcal{C}_{j}=F_{j} \star \mathcal{C} \text { and } \mathcal{T}_{s, j}=F_{j} \star \mathcal{T}_{s},
$$

and, for each $j$, considering

$$
f_{s, j}=\mathcal{C}_{j}+\mathcal{T}_{s, j}
$$

For each $j$, we assume that $f_{s, j}$ is known to us, and we aim to compute $\mathcal{C}_{j}$ and $\mathcal{T}_{s, j}$ from it. By the choice of the filter, we can then derive $\mathcal{C}$ and $\mathcal{T}_{s}$ from those.

1.5. Frequency Matching. In order to set up the separation problem in a reasonable way, the two filtered components $\mathcal{C}_{j}$ and $\mathcal{T}_{s, j}$ have to be comparable as we go to finer scales, so that the ratio of energies is more or less independent of scale. This ensures that separation is challenging at every scale. Thus we need to compute the norms $\left\|\mathcal{C}_{j}\right\|_{2}$ and $\left\|\mathcal{T}_{s, j}\right\|_{2}$, and then link $s$ to $2^{j}$ in such a way that both quantities are comparable. This will then also allow us to drive an asymptotic analysis based on the scale $j$ - upon which the size $s$ then depends.

Computing the asymptotic behavior of the norm of our filtered cartoon model, we face the problem that the behavior of $\hat{\mathcal{C}}$ is not always the same asymptotically for all possible elements. To resolve this problem, we restrict the model slightly to cartoons which satisfy

$$
|\hat{\mathcal{C}}(\xi)| \sim|\xi|^{-3 / 2}, \quad \xi \in \mathbb{R}^{2}
$$

We wish to note that this is only a very mild restriction, since for the boundary of the sphere $\mathcal{C}=S^{2}$, it can be proven that this condition is satisfied (see, e.g., [6]). In general, Randol [48] 
(cf. also [35]) proved, for instance, that if $\mathcal{C} \in C^{4}$ and if the Gaussian curvature is non-zero in every point of $\partial \mathcal{C}$, then $|\hat{\mathcal{C}}(\xi)| \leq C \cdot|\xi|^{-3 / 2}, \xi \in \mathbb{R}^{2}$.

With this additional assumption, we can now derive the following lemma whose proof is outsourced to Subsection 4.1.

\section{Lemma 1.1.}

$$
\left\|\mathcal{C}_{j}\right\|_{2}^{2} \sim 2^{-j}, \quad j \rightarrow \infty .
$$

We next compute the asymptotic behavior of the norm of our filtered texture model and also refer to Subsection 4.1 for the proof.

\section{Lemma 1.2.}

$$
\left\|\mathcal{T}_{s, j}\right\|_{2}^{2} \sim \sum_{m, \tilde{m}} e^{-\frac{|m-\tilde{m}|}{2}} \sum_{n \in \mathbb{Z}^{2} \cap \mathcal{A}_{s, j}} d_{m, n} \overline{d_{\tilde{m}, n}},
$$

where

$$
\mathcal{A}_{s, j}=\left\{\xi \in \mathbb{R}^{2}: \frac{2^{j-1}}{s} \leq|\xi| \leq \frac{2^{j+1}}{s}\right\} .
$$

The energy matching condition on $s$ and $j$ can now be derived from Lemmata 1.1 and 1.2 by choosing $s=s_{j}$ to satisfy

$$
\sum_{m, \tilde{m}} e^{-\frac{|m-\tilde{m}|}{2}} \sum_{n \in \mathbb{Z}^{2} \cap \mathcal{A}_{s_{j}, j}} d_{m, n} \overline{d_{\tilde{m}, n}}=2^{-j} .
$$

We now rewrite (2) as

$$
f_{j}=\mathcal{C}_{j}+\mathcal{T}_{j}
$$

and also simply write $\mathcal{A}_{j}$ instead of $\mathcal{A}_{s_{j}, j}$.

For illustrative purposes we present the energy matching condition for one particular sequence $\left(d_{m, n}\right)_{m, n \in \mathbb{Z}^{2}}$.

Example 1.1. If we exemplarily choose

$$
\left|d_{m, n}\right| \sim|m|^{-(2+\delta)} \cdot|n|^{-(2+\delta)}, \quad \delta>0,
$$

and observe that for the asymptotics we can ignore the 'side terms' $m \neq \tilde{m}$, the energy matching condition (4) becomes

$$
2^{-j} \sim \sum_{m}|m|^{-(4+2 \delta)} \cdot \sum_{n \in \mathbb{Z}^{2} \cap \mathcal{A}_{j}}|n|^{-(4+2 \delta)} .
$$

Now $\sum_{m}|m|^{-(4+2 \delta)}$ is constant, and since

$$
\int_{0}^{2 \pi} \int_{\frac{2^{j-1}}{s_{j}}}^{\frac{2^{j+1}}{s_{j}}} r^{-(4+2 \delta)} \cdot r d r d \varphi \sim\left(\frac{2^{j}}{s_{j}}\right)^{-2(1+\delta)}
$$

from (6), we finally obtain the condition $2^{-j} \sim\left(2^{j} / s_{j}\right)^{-2(1+\delta)}$, hence we can choose $s_{j}$ as

$$
s_{j}=2^{\frac{1+2 \delta}{2+2 \delta} j} .
$$

Thus, in particular, we have $s_{j}=\omega\left(2^{j / 2}\right)$ and $s_{j}=o\left(2^{j}\right)$ in this case. This implies that energy matching is achieved, if the size of a repeated patch of the texture, i.e., $1 / s_{j}$, is 
'slightly' larger than the thickness of the filtered boundary of the cartoon, more precisely, between $2^{-j}$ and $2^{-j / 2}$. Hence intuitively separation seems doubtful in this case. It will thus be surprising that in Corollary 1.1, we will show arbitrarily precise separation with the scale $j \rightarrow \infty$, if only $\delta>1$.

1.6. Separation via $\ell_{1}$ Minimization. In our analysis we aim at exploring the geometrical contents and, in particular, their difference of the cartoonlike structure $\mathcal{C}$ and texturelike structure $\mathcal{T}_{s}$. In [19], we have introduced a fundamental notion which allows to derive estimates on the accuracy of $\ell_{1}$ based separation of morphological objects. It was therein applied to prove that, given an image composed of pointlike and curvelike structures at all sufficiently fine scales, nearly-perfect separation can be achieved. Here we are concerned with a different situation, since such an asymptotical result is not possible, simply due to the fact that Gabor systems don't have a scaling parameter. Although our analysis will therefore be different in nature, the common bracket with [19] is again the utilization of the geometry of the components. This allows us to apply some of the novel techniques developed in [19] also in the setting considered in this paper. For the convenience of the reader, we will briefly recall those in this subsection.

Suppose we have two tight frames $\Phi_{1}, \Phi_{2}$ in a Hilbert space $\mathcal{H}$, and a signal vector $S=S_{1}^{0}+S_{2}^{0} \in \mathcal{H}$. We then consider the following optimization problem

(Sep) $\quad\left(S_{1}^{\star}, S_{2}^{\star}\right)=\operatorname{argmin}_{S_{1}, S_{2}}\left\|\Phi_{1}^{T} S_{1}\right\|_{1}+\left\|\Phi_{2}^{T} S_{2}\right\|_{1}$ subject to $S=S_{1}+S_{2}$.

We wish to remark that intentionally the norm is placed on the analysis coefficients rather than on the synthesis coefficients as in Basis Pursuit [9] to avoid self-terms in the frame setting. Hence we might not recover the most sparsest expansion, but it will turn out that this expansion is nevertheless sufficient for component separation. Also it is algorithmically not feasible to optimize over all possible expansions. In many studies of $\ell_{1}$ optimization, one then considers the mutual coherence

$$
\mu(\Phi, \Psi)=\max _{j} \max _{i}\left|\left\langle\phi_{i}, \psi_{j}\right\rangle\right|,
$$

whose importance was shown by [18]. This might be thought of as singleton coherence. In contrast, to exploit the knowledge of the geometrical structure of the components - more precisely, the location of the 'large' coefficients of their frame expansions -, in [19] the notion of cluster coherence was introduced, which bounds coherence between a single member of a frame $\Psi$ and a cluster of members of a second frame $\Phi$, clustered at $\mathcal{S}$.

Definition 1.1. Given tight frames $\Phi=\left(\phi_{i}\right)_{i}$ and $\Psi=\left(\psi_{j}\right)_{j}$ and an index subset $\mathcal{S}$ associated with expansions in frame $\Phi$, we define the cluster coherence

$$
\mu_{c}(\mathcal{S}, \Phi ; \Psi)=\max _{j} \sum_{i \in \mathcal{S}}\left|\left\langle\phi_{i}, \psi_{j}\right\rangle\right| .
$$

With this notion at hand, the following estimate concerning the accuracy of the extraction of $S_{1}^{0}$ and $S_{2}^{0}$ from the signal $S$ holds true:

Proposition 1.1. [19, Props. $1+2]$ Suppose that $S$ can be decomposed as $S=S_{1}^{0}+S_{2}^{0}$ so that each component $S_{i}^{0}$ is relatively sparse in $\Phi_{i}, i=1,2$, i.e.,

$$
\left\|1_{\mathcal{S}_{1}^{c}} \Phi_{1}^{T} S_{1}^{0}\right\|_{1}+\left\|1_{\mathcal{S}_{2}^{c}} \Phi_{2}^{T} S_{2}^{0}\right\|_{1} \leq \delta .
$$


Let $\left(S_{1}^{\star}, S_{2}^{\star}\right)$ solve (8). Then

$$
\left\|S_{1}^{\star}-S_{1}^{0}\right\|_{2}+\left\|S_{2}^{\star}-S_{2}^{0}\right\|_{2} \leq \frac{2 \delta}{1-2 \mu_{c}},
$$

where

$$
\mu_{c}=\max \left(\mu_{c}\left(\mathcal{S}_{1}, \Phi_{1} ; \Phi_{2}\right), \mu_{c}\left(\mathcal{S}_{2}, \Phi_{2} ; \Phi_{1}\right)\right) .
$$

The concepts of this section will now be applied to (SEP), at scale $j$ only. For this, the tight frames are $\Phi_{1}$, the full curvelet tight frame, and $\Phi_{2}$, the full Gabor tight frame, and $S$ is our filtered observed image $f_{j}$, which satisfies (cf. (5)),

$$
f_{j}=\mathcal{C}_{j}+\mathcal{T}_{j} .
$$

We apply the optimization problem (SEP), getting subsignal components $S_{1}^{\star}$ and $S_{2}^{\star}$, which we then relabel as the curvelet component $C_{j}$ and Gabor component $T_{j}$.

The cluster of indices of both, sparse approximation error and cluster coherence, depends on the choice of the cluster indices $\mathcal{S}_{1}$ - now denoted by $\mathcal{S}_{1, j}$ - for the curvelet system and the cluster indices $\mathcal{S}_{2}$ - now denoted by $\mathcal{S}_{2, j}$ - for the Gabor system. The sparse approximation error $\delta$ and the cluster coherence $\mu_{c}$ - let us remind the reader that these are merely analysis tools and are not part of the $\ell_{1}$ minimization procedure - relevant for a scale $j$ will then be denoted by

$$
\delta_{1, j} \quad \text { and } \quad \delta_{2, j}
$$

for relative sparsity for the cartoon and texture part, respectively, as well as

$$
\left(\mu_{c}\right)_{1, j}=\mu_{c}\left(\mathcal{S}_{1, j},\left\{\gamma_{\eta}\right\} ;\left\{\left(g_{s}\right)_{\lambda}\right\}\right) \text { and }\left(\mu_{c}\right)_{2, j}=\mu_{c}\left(\mathcal{S}_{2, j},\left\{\left(g_{s}\right)_{\lambda}\right\} ;\left\{\gamma_{\eta}\right\}\right)
$$

for the cluster coherence for a cluster of curvelets and Gabor elements, respectively.

1.7. Asymptotic Separation Result. Our aim is an asymptotic separation result for the relative $L^{2}$-error of the purported cartoon part $C_{j}$ and texture part $T_{j}$ decays to zero as $j \rightarrow \infty$. By Proposition 1.1, this follows if the clusters $\mathcal{S}_{1, j}$ and $\mathcal{S}_{2, j}$ can be chosen such that

$$
\max \left(\delta_{1, j}, \delta_{2, j}\right)=o\left(2^{-j / 2}\right), \quad \text { as } j \rightarrow \infty,
$$

and

$$
\max \left(\left(\mu_{c}\right)_{1, j},\left(\mu_{c}\right)_{2, j}\right) \rightarrow 0, \quad \text { as } j \rightarrow \infty .
$$

Our main result shows that this is indeed possible. For this, the set of significant coefficients $\mathcal{S}_{2, j}$ for $\mathcal{T}_{j}$ is defined by

$$
\mathcal{S}_{2, j}=B\left(0, r_{1, j}\right) \times B\left(0, r_{2, j}\right),
$$

for some $r_{1, j}, r_{2, j}>0$, where $B(0, r)$ shall denote the closed $\ell_{2}$ ball around the origin in $\mathbb{R}^{2}$.

Theorem 1.1. Assuming energy matching (4), we set

$$
\mathcal{A}_{j}=\left\{\xi \in \mathbb{R}^{2}: \frac{2^{j-1}}{s_{j}} \leq|\xi| \leq \frac{2^{j+1}}{s_{j}}\right\}
$$

and

$$
M_{a_{j}, \theta, s_{j}}=R_{\theta} \cdot s_{j}^{-1}\left(\operatorname{supp} \hat{\gamma}_{a_{j}, 0,0}+B_{1}(0,1)\right) \cap \mathbb{Z}^{2} .
$$


Suppose that there exist $r_{1, j}, r_{2, j}>0$ which satisfy

$$
\sum_{\substack{(m, n) \notin B\left(0, r_{1, j}\right) \times B\left(0, r_{2, j}\right) \\ n \in \mathcal{A}_{j}}} \sum_{\tilde{m} \in \mathbb{Z}^{2}}\left|d_{\tilde{m}, n}\right| e^{-\frac{|\tilde{m}-m|}{2}}=o\left(2^{-j / 2}\right), \quad j \rightarrow \infty
$$

and

(i) in case $s_{j}=o\left(2^{j}\right)$ as $j \rightarrow \infty$,

$$
\left|B\left(0, r_{2, j}\right) \cap M_{a_{j}, 0, s_{j}} \cap \mathbb{Z}^{2}\right|=o\left(2^{3 j / 4} \cdot s_{j}^{-1}\right), \quad \text { as } j \rightarrow \infty,
$$

(ii) as well as in case $s_{j}=\Omega\left(2^{j}\right)$ as $j \rightarrow \infty$,

$$
\left|B\left(0, r_{1, j}\right) \cap \mathbb{Z}^{2}\right|=o\left(2^{-3 j / 4} \cdot s_{j}\right), \quad \text { as } j \rightarrow \infty .
$$

Then we have asymptotically near-perfect separation:

$$
\frac{\left\|C_{j}-\mathcal{C}_{j}\right\|_{2}+\left\|T_{j}-\mathcal{T}_{j}\right\|_{2}}{\left\|\mathcal{C}_{j}\right\|_{2}+\left\|\mathcal{T}_{j}\right\|_{2}} \rightarrow 0, \quad j \rightarrow \infty
$$

Most interesting is the consideration of the special case already focussed on in Example 1.1. In this situation asymptotically near-perfect separation is always attained provided that $\delta>1$. The precise statement is the following

Corollary 1.1. Suppose that the sequence $\left(d_{m, n}\right)_{m, n \in \mathbb{Z}^{2}}$ satisfies

$$
\left|d_{m, n}\right| \sim|m|^{-(2+\delta)} \cdot|n|^{-(2+\delta)}, \quad \delta>1 .
$$

Then we have asymptotically near-perfect separation:

$$
\frac{\left\|C_{j}-\mathcal{C}_{j}\right\|_{2}+\left\|T_{j}-\mathcal{T}_{j}\right\|_{2}}{\left\|\mathcal{C}_{j}\right\|_{2}+\left\|\mathcal{T}_{j}\right\|_{2}} \rightarrow 0, \quad j \rightarrow \infty
$$

Theorem 1.1 and Corollary 1.1 are both proved in Subsection 4.1.

1.8. Interpretation. To heuristically understand why cartoon and texture can be separated at all, consider the worst case scenario that an image is composed of one cartoon and a periodized cartoon as is illustrated in Figure 2.

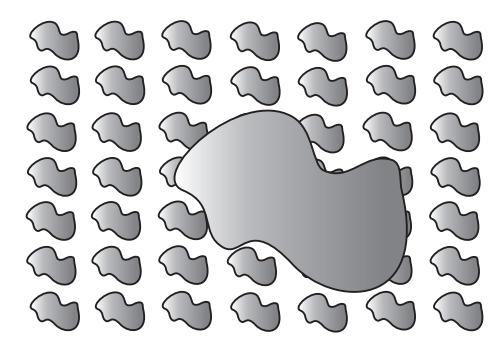

Figure 2. Periodic small cartoons versus one large cartoon.

The periodic cartoon is then of the form

$$
f=\sum_{k \in \mathbb{Z}^{2}} 1_{C}(x+k), \quad C \subset \mathbb{R}^{2} .
$$


Taking the Fourier transform, we observe that

$$
\hat{f}=\hat{1}_{C} \cdot \sum_{k \in \mathbb{Z}^{2}} e^{2 \pi i\langle k, \xi\rangle}
$$

with the second part being responsible for the sparsity in a Gabor system. This sparsity is the key for separation from a single 'large' cartoon. A further question for this particular situation might be: Given a set $C$ and a lattice $A \mathbb{Z}^{2}$, how sparse is the corresponding periodization? However, this goes far beyond our model, wherefore we do not treat this question here, but label it an interesting direction for future research.

Let us now take a closer look at Corollary 1.1. To first build up intuition on the energy balancing condition, we consider a curvelet of scale $j$ and a Gabor element of size $s$. If $s=s_{j}$ satisfies the energy balancing condition (7), the spatial footprint of the Gabor element is about the size of the curvelet, since $s_{j}=\omega\left(2^{j / 2}\right)$ and $s_{j}=o\left(2^{j}\right)$ in this case, which is illustrated in Figure 3.

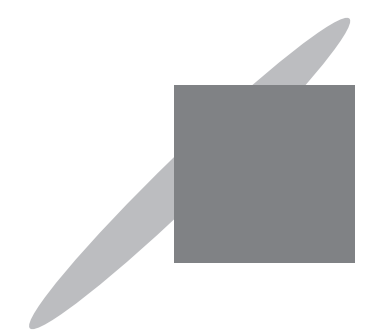

FigurE 3. The essential spatial support of a curvelet $\gamma_{j, k, \ell}$ and Gabor element $\left(g_{s}\right)_{m, n}$ satisfying the energy balancing condition $s=s_{j}$ with $s_{j}$ defined in (7).

This also visualizes the relation between the filtered cartoon part $\mathcal{C}_{j}$ and the filtered texture part $\mathcal{T}_{j}\left(=\mathcal{T}_{s_{j}, j}\right)$, since those will in fact be similarly connected. It is intuitively clear that this is the 'worst scenario'. It seems at first sight quite astonishing that separation is still possible in this case. However, the geometrical clustering of the significant coefficients solves the mystery, and the notion of cluster coherence makes this precise. In fact, our result shows that the whole cluster of Gabor elements $\left(g_{s_{j}}\right)_{m, n}$ with $(m, n) \in B\left(0, r_{1, j}\right) \times B\left(0, r_{2, j}\right)$ cannot be 'glued together' so that it generates a single curvelet. The same is true for the whole cluster of significant curvelets, which are unable to generate a single Gabor element. To visualize this would require to show the footprints of curvelets and Gabor elements in a 5 dimensional 'phase space': Spatial domain $\mathbb{R}^{2}$, orientation $[0,2 \pi]$, and frequency $\mathbb{R}^{2}$.

1.9. Extensions. Theorem 1.1 is amenable to the following generalizations and extensions.

- Thresholding. Thresholding is a separation strategy, which is commonly utilized as a substitute for $\ell_{1}$ minimization due to the fact that its algorithmic complexity is much lower. Hence one might ask: Can we derive a similar asymptotic separation result when using thresholding? And in fact, this is true. We though decided not to include the whole analysis, since it would go beyond the scope of this paper. We would just like to mention that similar techniques as in [39] are employable, but the technical details, which will now in addition require the special treatment of the scale of curvelets and the window size of Gabor atoms, will be very tedious. 
- Intersections. Natural images are typically composed not only of one smoothly filled $C^{2}$ edge curve, but several with presumably various intersections. In a similar way as it is commented upon in [5], we can also argue here that intersections will not affect our analysis. Thus the framework can be extended to separating several cartoon-like objects from texture.

- Other Systems. Theorem 1.1 holds without change for many other pairs of frames and bases, such as, e.g., by [40], for the pair or shearlets (cf. [31, 42, 37, 41]) and Gabor systems.

- Noise. Theorem 1.1 is resilient to noise impact; an image composed of $\mathcal{C}$ and $\mathcal{T}$ with additive 'sufficiently small' noise exhibits the same asymptotic separation. This can be easily deduced by utilizing ideas from [19, Sect. 8].

\section{Relative Sparsity}

2.1. Cartoon. Let $\mathcal{C}_{j}$ be the filtered version of the cartoon-part $\mathcal{C}$ of the considered image, where - as detailed in Subsection 1.4 - we use $\hat{F}_{j}(\xi)=W\left(a_{j}|\xi|\right)$ as a filter. We now describe how to partition $\mathcal{C}_{j}$ into smaller pieces which will then in a second step be bent to 'line objects' separately. The first part is inspired by a technique employed in [5], whereas the second part follows ideas introduced in [19].

We start by first smoothly localizing $\mathcal{C}_{j}$ near dyadic squares with a prescribed radius, which is chosen such that the curvature of each piece is controllable, but independent on $j$. More precisely, we define a partition of unity $\left(w_{Q}\right)_{Q \in \mathcal{Q}}$ with those properties, and let

$$
\mathcal{C}_{j, Q}=\mathcal{C}_{j} \cdot w_{Q}
$$

We would like the reader to notice that the number of pieces $\mathcal{C}_{j, Q}$ is finite and independent on $j$. Also there is no need to consider those $\mathcal{C}_{j, Q}$ which are smooth, since their associated curvelet coefficients have sufficient decay. For each of the remaining functions $\mathcal{C}_{j, Q}$, without loss of generality we can assume - as was done in [5, Sect. 6.1] - that the edge discontinuity is centered at 0 and that its first derivative equals zero pointing in the vertical direction. Hence, WLOG, the splitted cartoon might take a form as illustrated on the LHS of Figure 4.

Having partitioned the filtered cartoon $\mathcal{C}_{j}$, we now apply a diffeomorphism $\phi_{Q}$ to each piece $\mathcal{C}_{j, Q}$, which equals the identity outside of a compact set, thereby straightening out the discontinuity. For a similar strategy, we would like to refer the reader to [19, Sect. 7]. The resulting piece can now be modeled as

$$
H_{j, Q}=\left(H \star F_{j}\right) \cdot v_{Q}
$$

where $H=1_{x_{1} \geq 0}$ is the heaviside function and $v_{Q}$ is a $C^{\infty}$-function supported in a dyadic square of sidelength $\rho$, say. An illustration of this slitting and bending is presented in Figure 4.

We then require the following lemma, which is stated slightly more general than needed. For this, we let $H^{\nu}$ be defined by

$$
\left\langle\hat{H}^{\nu}, f\right\rangle=\int\left|\xi_{1}\right|^{-\nu} f\left(\xi_{1}, 0\right) d \xi
$$




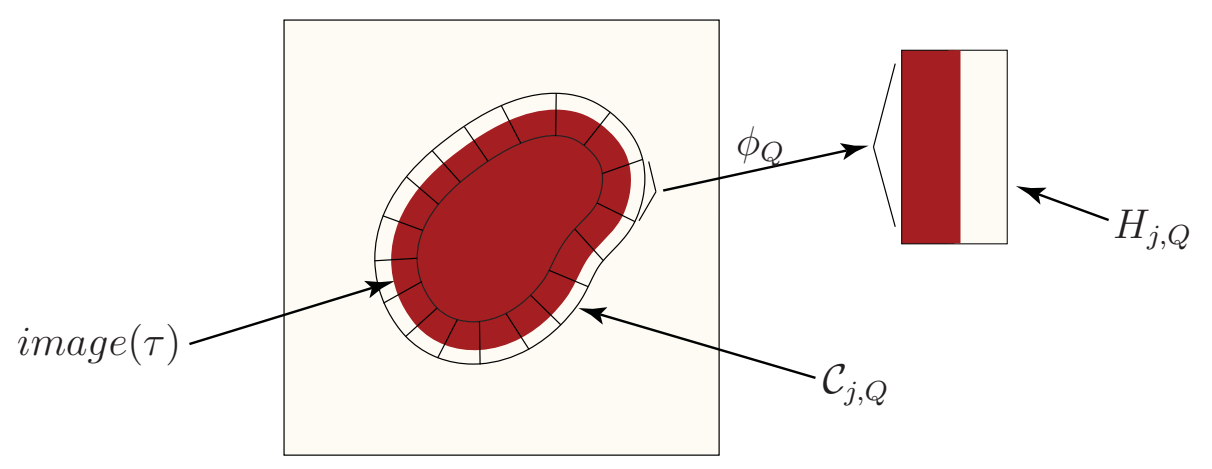

FigurE 4 . The filtered cartoon $\mathcal{C}_{j}$ and the mapping $\phi_{Q}: \mathcal{C}_{j, Q} \rightarrow H_{j, Q}$.

hence, in particular, $H^{1}=H$.

Lemma 2.1. For each $N=1,2, \ldots$, there is a constant $c_{N}$ so that

$$
\left|\left\langle\left(H^{\nu} \star F_{j}\right) \cdot v_{Q}, \gamma_{a, b, \theta}\right\rangle\right| \leq c_{N} \cdot a^{\nu-1 / 4} \cdot 1_{\left|\log _{2}\left(a / a_{j}\right)\right|<3} \cdot\left\langle\frac{|\sin (\theta)-1|}{a}\right\rangle^{-N} \cdot\left\langle\left|b_{1} / a\right|\right\rangle^{-N} .
$$

Proof. First, we observe that

$$
\left|\left\langle\left(H^{\nu} \star F_{j}\right) \cdot v_{Q}, \gamma_{a, b, \theta}\right\rangle\right| \leq c_{a} 1_{\left|\log _{2}\left(a / a_{j}\right)\right|<3} .
$$

Therefore, we now restrict to the case $a=a_{j}$. Next, using Parseval, and letting $\omega(\xi)$ denote the angular component of $\xi$,

$$
\begin{aligned}
\left\langle\left(H^{\nu} \star F_{j}\right) \cdot v_{Q}, \gamma_{a, b, \theta}\right\rangle & \\
= & \left\langle\hat{H}^{\nu} \hat{F}_{j}, \hat{v}_{Q} \star \hat{\gamma}_{a, b, \theta}\right\rangle \\
= & c \cdot \int\left|\xi_{1}\right|^{-\nu} \int \hat{v}_{Q}(\tau) a^{3 / 4} W\left(a\left|\xi_{1}-\tau\right|\right) V\left(\left(\omega\left(\xi_{1}-\tau\right)-\theta\right) / \sqrt{a}\right) e^{-i\left\langle b, \xi_{1}-\tau\right\rangle} d \tau \\
& \cdot W\left(a\left|\xi_{1}\right|\right) d \xi_{1} .
\end{aligned}
$$

By definition, the function $\hat{v}_{Q}$ is essentially supported in a square of radius $\rho^{-1}$. Hence we approximately have $\xi_{1}-\tau \subseteq\left\{\xi:\left|\xi_{2}\right| \leq \rho^{-1}\right\}$. Assuming this condition, if $\theta \neq \pi / 2$, then, for sufficiently small $a$,

$$
W\left(a\left|\xi_{1}-\tau\right|\right) V\left(\left(\omega\left(\xi_{1}-\tau\right)-\theta\right) / \sqrt{a}\right)=0 \quad \text { for all } \xi_{1}, \tau .
$$

Thus, we can conclude that

$$
\left|\left\langle\left(H^{\nu} \star F_{j}\right) \cdot v_{Q}, \gamma_{a, b, \theta}\right\rangle\right| \leq\left\langle\frac{|\sin (\theta)-1|}{a}\right\rangle^{-N}
$$

Now, continuing the computation in (10) by using a change of variables, we obtain

$$
\begin{aligned}
& \left\langle\left(H^{\nu} \star F_{j}\right) \cdot v_{Q}, \gamma_{a, b, \theta}\right\rangle \\
& =c \cdot a^{\nu-1 / 4} \cdot \int\left|\xi_{1}\right|^{-\nu} \int \hat{v}_{Q}(\tau) W\left(\left|\xi_{1}-a \tau\right|\right) V\left(\left(\omega\left(a^{-1} \xi_{1}-\tau\right)-\theta\right) / \sqrt{a}\right) e^{i\langle b, \tau\rangle} d \tau \\
& \quad \cdot W\left(\left|\xi_{1}\right|\right) e^{-i\left(b_{1} / a\right) \xi_{1}} d \xi_{1} .
\end{aligned}
$$


Since $W$ is compactly supported, the size of the support of the function $\hat{G}_{a}$, defined by

$$
\hat{G}_{a}\left(\xi_{1}\right)=a^{\nu-1 / 4} \cdot\left|\xi_{1}\right|^{-\nu} \int \hat{v}_{Q}(\tau) W\left(\left|\xi_{1}-a \tau\right|\right) V\left(\left(\omega\left(a^{-1} \xi_{1}-\tau\right)-\theta\right) / \sqrt{a}\right) e^{i\langle b, \tau\rangle} d \tau W\left(\left|\xi_{1}\right|\right),
$$

is independent of $a$. Using repeated partial integration, we conclude that

$$
|G(b)| \leq\left\|\hat{G}_{a}\right\|_{\infty} \cdot\left\langle\left|b_{1} / a\right|\right\rangle^{-N} \leq a^{\nu-1 / 4} \cdot\left\langle\left|b_{1} / a\right|\right\rangle^{-N} .
$$

The result is then completed by invoking (9) and (11).

Let us now introduce the cluster of significant curvelet coefficients for $\mathcal{C}_{j}$. First, we define clusters for the manipulated pieces $H_{j, Q}$, which are then 'lifted' to a cluster for $\mathcal{C}_{j}$. Intuitively, the cluster around $H_{j, Q}$ should spatially contain a carefully growing neighborhood of the discontinuity $\{0\} \times[-\rho, \rho]$ and directionally contain again a carefully growing neighborhood now of the direction of the discontinuity. For this, measuring the spatial distances between a point $x$ and a set $A$ with

$$
d_{2}(x, A)=\min _{a \in A}\|x-a\|_{2}, \quad x \in \mathbb{R}^{2}, A \subseteq \mathbb{R}^{2},
$$

we define the neighborhood of the discontinuity $\{0\} \times[-\rho, \rho]$ in phase space by

$$
\mathcal{N}_{2}^{P S}\left(a_{j}\right)=\left\{b \in \mathbb{R}^{2}: d_{2}(b,\{0\} \times[-2 \rho, 2 \rho]) \leq a_{j}^{1-\varepsilon}\right\} \times\left[0, \sqrt{a_{j}}\right],
$$

where $\varepsilon \in\left(0, \frac{1}{8}\right)$. We are then led to define its corresponding neighborhood for discrete parameter sets - setting $\theta_{j, \ell}=\pi \ell / 2^{j / 2}$ and $b_{j, k, \ell}=R_{\theta_{j, \ell}} D_{2^{-j}} k$ - by

$$
\tilde{\mathcal{S}}_{j}=\left\{(j, k, \ell):\left(b_{j, k, \ell}, \theta_{j, \ell}\right) \in \mathcal{N}_{2}^{P S}\left(a_{j}\right)\right\} .
$$

In order to 'lift' these clusters to a cluster for $\mathcal{C}_{j}$, we will make use of the filtering matrix associated with the filter $F_{j}$, i.e., of the matrix $M_{F_{j}}=\left(\left\langle\gamma_{\eta}, F_{j} \star \gamma_{\eta^{\prime}}\right\rangle\right)_{\eta, \eta^{\prime}}$. We next need to recall that the action of a diffeomorphism $\phi_{Q}$ on a distribution $f$ by $\phi_{Q}^{\star} f=f \circ \phi_{Q}$ induces a linear transformation on the space of curvelet coefficients. With $\alpha(f)$ the curvelet coefficients of $f$ and $\beta(f)$ the curvelet coefficients of $\phi_{Q}^{\star} f$, we obtain a linear operator $M_{\phi_{Q}}$ defined by

$$
M_{\phi_{Q}}(\alpha(f))=\beta(f) .
$$

Letting now $t_{\eta^{\prime}, n_{j}^{2}}$ denote the amplitude of the $n_{j}^{2}$ 'th largest element of the $\eta^{\prime \prime}$ th column of the matrix $M_{F_{j}} \cdot M_{\left(\phi_{Q}\right)^{-1}}$, we can define the overall cluster set for $\mathcal{C}_{j}$ by

$$
\mathcal{S}_{1, j}=\bigcup_{Q} \mathcal{S}_{1, j, Q}
$$

where

$$
\mathcal{S}_{1, j, Q}=\left\{\eta: \eta^{\prime} \in \tilde{\mathcal{S}}_{j} \text { and }\left|M_{F_{j}} \cdot M_{\left(\phi_{Q}\right)^{-1}}\left(\eta, \eta^{\prime}\right)\right|>t_{\eta^{\prime}, n_{j}^{2}}\right\} .
$$

The following result will be needed for estimating the relative sparsity of each $\mathcal{C}_{j}$ with respect to the just defined cluster $\mathcal{S}_{1, j}$.

Lemma 2.2. Let $\alpha_{j}=\left(\left\langle H_{j, Q}, \gamma_{\eta}\right\rangle\right)_{\eta}$ denote the curvelet frame coefficients of $H_{j, Q}=(H \star$ $\left.F_{j}\right) \cdot v_{Q}$. Then for all sufficiently large $j$,

$$
\left\|\alpha_{j}\right\|_{p} \leq c_{p} \cdot 2^{j(1 /(2 p)-3 / 4)}, \quad \forall p>0 .
$$


Proof. This proof follows the lines of the proof of [19, Lem. 6.2] very closely, wherefore we decided not to state it explicitly. It should just be mentioned that here we use Lemma 2.1 instead of [19, Lem. 6.1], which leads to a difference in the asymptotic behavior by $2^{-j}$.

The decay estimate of the cluster approximate error $\delta_{1, j}$ is then given by the following result.

Lemma 2.3.

$$
\delta_{1, j}=\sum_{\eta \in \Delta \backslash \mathcal{S}_{1, j}}\left|\left\langle\gamma_{\eta}, \mathcal{C}_{j}\right\rangle\right|=o\left(2^{-j / 2}\right), \quad j \rightarrow \infty .
$$

Proof. Since the proof is very similar to [19, Lem. 8.5], we will not state it, but merely mention that instead of [19, Lem. 6.2] we here apply Lemma 2.2.

2.2. Texture. The decay estimate of the cluster approximation error $\delta_{2, j}$ is given by the following result.

Lemma 2.4. If there exist $r_{1, j}, r_{2, j}>0$ such that

$$
\sum_{\substack{(m, n) \notin B\left(0, r_{1, j}\right) \times B\left(0, r_{2, j}\right) \\ n \in \mathcal{A}_{j}}} \sum_{\tilde{m} \in \mathbb{Z}^{2}}\left|d_{\tilde{m}, n}\right| e^{-\frac{|\tilde{m}-m|}{2}}=o\left(2^{-j / 2}\right), \quad j \rightarrow \infty,
$$

then

$$
\delta_{2, j}=o\left(2^{-j / 2}\right), \quad j \rightarrow \infty .
$$

Proof. First,

$$
\delta_{2, j}=\sum_{\left.(m, n) \notin B\left(0, r_{1, j}\right)\right) \times B\left(0, r_{2, j}\right.}\left|\left\langle\left(g_{s_{j}}\right)_{m, n}, \mathcal{T}_{j}\right\rangle\right| .
$$

Similar to the proof of Lemma 1.2, we have

$$
\left|\left\langle\left(g_{s_{j}}\right)_{m, n}, \mathcal{T}_{j}\right\rangle\right| \sim 1_{\mathcal{A}_{j}}(n) \cdot\left|\sum_{\tilde{m} \in \mathbb{Z}^{2}} d_{\tilde{m}, n} e^{-\frac{|\tilde{m}-m|}{2}}\right|, \quad j \rightarrow \infty .
$$

This immediately implies the claim.

To derive a better understanding, we analyze again the situation examined in Example 1.1.

Example 2.1. We here consider the situation that

$$
\left|d_{m, n}\right| \sim|m|^{-(2+\delta)} \cdot|n|^{-(2+\delta)}, \quad \delta>0,
$$

We aim to derive conditions on $r_{1, j}, r_{2, j}>0$ such that (12) is satisfied, i.e., such that

$$
T_{1} \cdot T_{2}+T_{3} \cdot T_{4}=o\left(2^{-j / 2}\right), \quad j \rightarrow \infty,
$$


where

$$
\begin{aligned}
T_{1} & =\sum_{|m| \geq r_{1, j}}\left[C \cdot e^{-\frac{|m|}{2}}+\sum_{\tilde{m} \in \mathbb{Z}^{2} \backslash\{0\}}|\tilde{m}|^{-(2+\delta)} e^{-\frac{|\tilde{m}-m|}{2}}\right], \\
T_{2} & =\sum_{n \in \mathbb{Z}^{2} \cap \mathcal{A}_{j}}|n|^{-(2+\delta)}, \\
T_{3} & =\sum_{|m| \leq r_{1, j}}\left[C \cdot e^{-\frac{|m|}{2}}+\sum_{\tilde{m} \in \mathbb{Z}^{2} \backslash\{0\}}|\tilde{m}|^{-(2+\delta)} e^{-\frac{|\tilde{m}-m|}{2}}\right], \\
T_{4} & =\sum_{n \in \mathbb{Z}^{2} \cap \mathcal{A}_{j} \cap B\left(0, r_{2, j}\right)^{c}}|n|^{-(2+\delta)} .
\end{aligned}
$$

Firstly,

$$
C \cdot \int_{r_{1, j}}^{\infty} e^{-\frac{r}{2}} \cdot r d r+\int_{|x| \geq r_{1, j}} \int_{|y| \geq 1}|y|^{-(2+\delta)} e^{-\frac{|x-y|}{2}} \sim r_{1, j}^{-\delta}
$$

hence

$$
T_{1} \sim r_{1, j}^{-\delta}
$$

Secondly,

$$
\int_{\frac{2^{j-1}}{s_{j}}}^{\frac{2^{j+1}}{s_{j}}} r^{-(2+\delta)} \cdot r d r \sim\left(\frac{2^{j}}{s_{j}}\right)^{-\delta}
$$

hence

$$
T_{2} \sim\left(\frac{2^{j}}{s_{j}}\right)^{-\delta}
$$

Thirdly,

$$
T_{3} \sim 1
$$

And, fourthly,

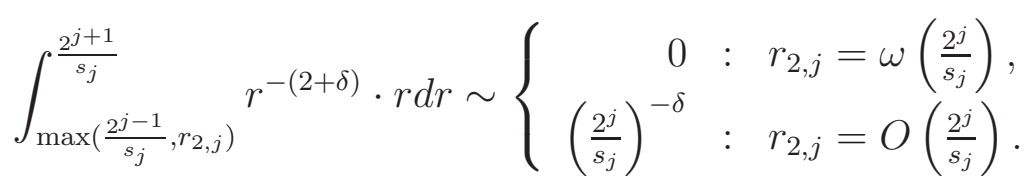

hence

$$
T_{4} \sim\left\{\begin{array}{rll}
0 & : & r_{2, j}=\omega\left(\frac{2^{j}}{s_{j}}\right) \\
\left(\frac{2^{j}}{s_{j}}\right)^{-\delta} & : & r_{2, j}=O\left(\frac{2^{j}}{s_{j}}\right) .
\end{array}\right.
$$

Applying (14)-(17) to (13) yields

$$
\delta_{1, j} \sim r_{1, j}^{-\delta} \cdot\left(\frac{2^{j}}{s_{j}}\right)^{-\delta}+\left\{\begin{array}{rll}
0 & : & r_{2, j}=\omega\left(\frac{2^{j}}{s_{j}}\right), \\
\left(\frac{2^{j}}{s_{j}}\right)^{-\delta}: & r_{2, j}=O\left(\frac{2^{j}}{s_{j}}\right) .
\end{array}\right.
$$


Taking the energy matching condition (7) from Example 1.1 into account,

$$
\delta_{1, j} \sim r_{1, j}^{-\delta} \cdot 2^{-\frac{\delta}{2+2 \delta} j}+\left\{\begin{array}{rll}
0 & : & r_{2, j}=\omega\left(\frac{2^{j}}{s_{j}}\right) \\
2^{-\frac{\delta}{2+2 \delta} j} & : & r_{2, j}=O\left(\frac{2^{j}}{s_{j}}\right) .
\end{array}\right.
$$

However, $2^{-\frac{\delta}{2+2 \delta} j}=\omega\left(2^{-j / 2}\right)$. Hence, condition (12) is only fulfilled, if and only if

$$
r_{1, j}=\omega\left(\frac{2^{j}}{s_{j}}\right)^{1 / \delta}=\omega\left(2^{\frac{1}{2 \delta(1+\delta)} j}\right) \quad \text { and } \quad r_{2, j}=\omega\left(\frac{2^{j}}{s_{j}}\right)=\omega\left(2^{\frac{1}{2(1+\delta)} j}\right) .
$$

\section{Cluster Coherence}

3.1. Interaction of Gabor Elements and Curvelets. We start by estimating an inner product of a Gabor element and a curvelet. Depending on how $s_{j}$ relates to $2^{j}$, we have to distinguish three cases. Note however, that the decay rate in all three cases is $O\left(2^{-j / 4}\right)$ as $j \rightarrow \infty$.

Lemma 3.1. For each $N=1,2, \ldots$, there is a constant $c_{N}$ so that,

(i) if $s_{j}=o\left(2^{j / 2}\right)$ as $j \rightarrow \infty$,

$$
\left|\left\langle\gamma_{a_{j}, b, \theta},\left(g_{s_{j}}\right)_{m, n}\right\rangle\right| \leq c_{N} \cdot 2^{-3 j / 4} \cdot s_{j} \cdot 1_{M_{a_{j}, \theta}, s_{j}}(n) \cdot\left\langle\left|s_{j} b-\frac{m}{2}\right|_{R_{\theta}}\right\rangle^{-N}
$$

(ii) if $s_{j}=\Omega\left(2^{j / 2}\right)$ and $s_{j}=o\left(2^{j}\right)$ as $j \rightarrow \infty$,

$$
\left|\left\langle\gamma_{a_{j}, b, \theta},\left(g_{s_{j}}\right)_{m, n}\right\rangle\right| \leq c_{N} \cdot 2^{-j / 4} \cdot 1_{M_{a_{j}, \theta}, s_{j}}(n) \cdot\left\langle\left|\left(s_{j} b_{1}-\frac{m_{1}}{2}, 2^{j / 2}\left(b_{2}-\frac{m_{2}}{2 s_{j}}\right)\right)\right|_{R_{\theta}}\right\rangle^{-N},
$$

(ii) if $s_{j}=\Omega\left(2^{j / 2}\right)$ and $s_{j}=o\left(2^{j}\right)$ as $j \rightarrow \infty$,

$$
\left|\left\langle\gamma_{a_{j}, b, \theta},\left(g_{s_{j}}\right)_{m, n}\right\rangle\right| \leq c_{N} \cdot 2^{3 j / 4} \cdot s_{j}^{-1} \cdot 1_{M_{a_{j}, \theta}, s_{j}}(n) \cdot\left\langle\left|b-\frac{m}{2 s_{j}}\right|_{D_{2^{j}} R_{\theta}}\right\rangle^{-N},
$$

where $M_{a_{j}, \theta, s_{j}}$ is defined as in Theorem 1.1.

Figure 5 illustrates the set $M_{a_{j}, \theta, s_{j}}$ for $\theta=0$, whereas the three cases in which Lemma 3.1 is split into are illustrated in Figure 6.

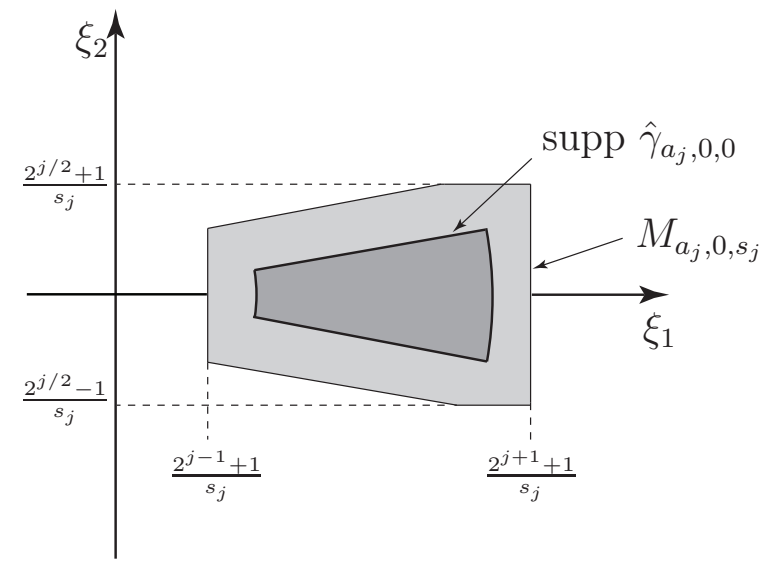

FiguRE 5. Support of the set $M_{a_{j}, 0, s_{j}}$. 


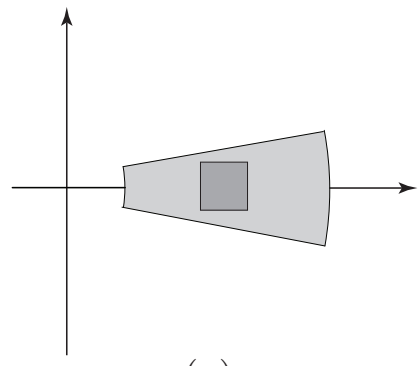

(a)

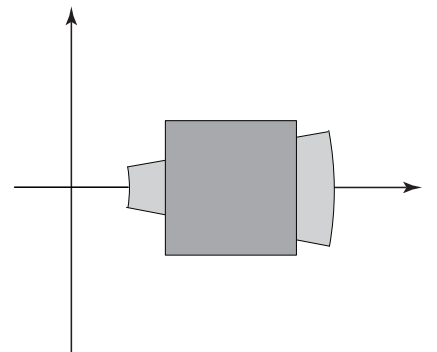

(b)

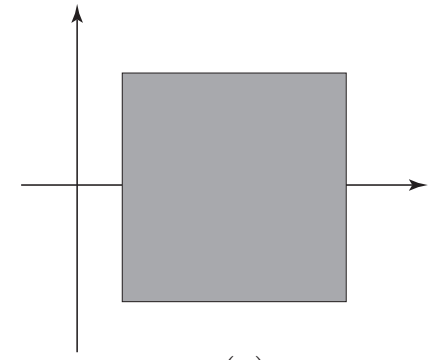

(c)

FiguRE 6. Relation between the support of a curvelet $\hat{\gamma}_{j, 0, k}$ and the support of a Gabor element $\left(\hat{g}_{s_{j}}\right)_{m,\left(n_{1}, 0\right)}$. (a): Case $s_{j}=o\left(2^{j / 2}\right)$. (b): Case $s_{j}=\Omega\left(2^{j / 2}\right)$ and $s_{j}=o\left(2^{j}\right)$. (c): Case $s_{j}=\Omega\left(2^{j}\right)$.

Proof of Lemma 3.1. First, by Plancherel and the definition of $M_{a_{j}, \theta, s_{j}}$,

$$
\begin{aligned}
& \left\langle\gamma_{a_{j}, b, \theta},\left(g_{s}\right)_{m, n}\right\rangle \\
& =c \cdot a_{j}^{3 / 4} \int W\left(a_{j}|\xi|\right) V\left((\omega-\theta) / \sqrt{a_{j}}\right) \hat{g}_{s_{j}}\left(\xi-s_{j} n\right) e^{i\left(b-\frac{m}{2 s_{j}}\right) \xi} d \xi \\
& =c \cdot a_{j}^{3 / 4} \cdot s_{j}^{-1} \cdot 1_{M_{a_{j}, \theta,} s_{j}}(n) \int W\left(a_{j}|\xi|\right) V\left((\omega-\theta) / \sqrt{a_{j}}\right) \hat{g}\left(\frac{\xi}{s_{j}}-n\right) e^{i\left(b-\frac{m}{2 s_{j}}\right) \xi} d \xi .
\end{aligned}
$$

It now remains to estimate

$$
T=\int W\left(a_{j}|\xi|\right) V\left((\omega-\theta) / \sqrt{a_{j}}\right) \hat{g}\left(\frac{\xi}{s_{j}}-n\right) e^{i\left(b-\frac{m}{2 s_{j}}\right) \xi} d \xi
$$

in the three cases the claim is split into. For this, in all three cases, WLOG we assume that $\theta=0$.

Case $s_{j}=o\left(2^{j / 2}\right)$ as $j \rightarrow \infty$. With $\zeta=\frac{\xi}{s_{j}}$, and letting $\omega(\cdot)$ denote the angular component,

$$
|T|=\left|s_{j}^{2} \cdot \int W\left(a_{j} s_{j}|\zeta|\right) V\left(\omega\left(s_{j} \zeta\right) / \sqrt{a_{j}}\right) \hat{g}(\zeta-n) e^{i\left(s_{j} b-\frac{m}{2}\right) \zeta} d \zeta\right| .
$$

Applying integration by parts, for any $k=1,2, \ldots$,

$$
\begin{aligned}
|T| & =s_{j}^{2} \cdot\left|s_{j} b-\frac{m}{2}\right|^{-k}\left|\int \Delta^{k}\left[W\left(a_{j} s_{j}|\zeta|\right) V\left(\omega\left(s_{j} \zeta\right) / \sqrt{a_{j}}\right) \hat{g}(\zeta-n)\right] e^{i\left(s_{j} b-\frac{m}{2}\right) \zeta} d \zeta\right| \\
& \leq s_{j}^{2} \cdot\left|s_{j} b-\frac{m}{2}\right|^{-k} \int\left|\Delta^{k}\left[W\left(a_{j} s_{j}|\zeta|\right) V\left(\omega\left(s_{j} \zeta\right) / \sqrt{a_{j}}\right) \hat{g}(\zeta-n)\right]\right| d \zeta .
\end{aligned}
$$

Hence

$$
\begin{aligned}
\left(1+\left|s_{j} b-\frac{m}{2}\right|^{k}\right) \cdot|T| \leq & \int\left|W\left(a_{j} s_{j}|\zeta|\right) V\left(\omega\left(s_{j} \zeta\right) / \sqrt{a_{j}}\right) \hat{g}(\zeta-n)\right| d \xi \\
& +\int\left|\Delta^{k}\left[W\left(a_{j} s_{j}|\zeta|\right) V\left(\omega\left(s_{j} \zeta\right) / \sqrt{a_{j}}\right) \hat{g}(\zeta-n)\right]\right| d \zeta .
\end{aligned}
$$


It can be shown that, for each $k$, there exists $c_{k}<\infty$ such that, for all $a_{j}$,

$$
\int\left|W\left(a_{j} s_{j}|\zeta|\right) V\left(\omega\left(s_{j} \zeta\right) / \sqrt{a_{j}}\right) \hat{g}(\zeta-n)\right| d \xi+\int\left|\Delta^{k}\left[W\left(a_{j} s_{j}|\zeta|\right) V\left(\omega\left(s_{j} \zeta\right) / \sqrt{a_{j}}\right) \hat{g}(\zeta-n)\right]\right| d \zeta \leq c_{k} .
$$

Further, for each $k=1,2, \ldots$,

$$
\left\langle\left|s_{j} b-\frac{m}{2}\right|\right\rangle^{k}=\left(1+\left|s_{j} b-\frac{m}{2}\right|^{2}\right)^{\frac{k}{2}} \leq \frac{k}{2}\left(1+\left|s_{j} b-\frac{m}{2}\right|^{k}\right),
$$

we obtain

$$
|T| \leq c_{N} \cdot s_{j}^{2} \cdot\left\langle\left|s_{j} b-\frac{m}{2}\right|\right\rangle^{-N}
$$

Reinserting $\theta$ and using (18), claim (i) is proved.

Case $s_{j}=\Omega\left(2^{j / 2}\right)$ and $s_{j}=o\left(2^{j}\right)$ as $j \rightarrow \infty$. With $\zeta=\left(s_{j}^{-1} \xi_{1}, 2^{-j / 2} \xi_{2}\right)=: \varphi_{j}(\xi)$,

$$
\begin{aligned}
|T|= & \mid 2^{j / 2} \cdot s_{j} \int W\left(a_{j}\left|\varphi_{j}^{-1}(\zeta)\right|\right) V\left(\omega\left(\varphi_{j}^{-1}(\zeta)\right) / \sqrt{a_{j}}\right) \hat{g}\left(\zeta_{1}-n_{1}, 2^{j / 2} s_{j}^{-1} \zeta_{2}-n_{2}\right) \\
& \cdot e^{i\left(s_{j} b_{1}-\frac{m_{1}}{2}, 2^{j / 2}\left(b_{2}-\frac{m_{2}}{2 s_{j}}\right)\right) \zeta} d \zeta \mid .
\end{aligned}
$$

Repeated integration by parts - similar as in the first case - implies

$$
|T| \leq c_{N} \cdot 2^{j / 2} \cdot s_{j} \cdot\left\langle\left|\left(s_{j} b_{1}-\frac{m_{1}}{2}, 2^{j / 2}\left(b_{2}-\frac{m_{2}}{2 s_{j}}\right)\right)\right|\right\rangle^{-N} .
$$

Reinserting $\theta$ and using (18), proves claim (ii).

Case $s_{j}=\Omega\left(2^{j}\right)$ as $j \rightarrow \infty$. With $\zeta=D_{2^{-j}} \xi$,

$$
|T|=\left|2^{3 j / 2} \int W(|\zeta|) V\left(\omega\left(D_{2^{j}} \zeta\right) / \sqrt{a_{j}}\right) \hat{g}\left(D_{2^{j}} \frac{\zeta}{s_{j}}-n\right) e^{i\left(b-\frac{m}{2 s_{j}}\right) D_{2^{j}} \zeta} d \zeta\right| .
$$

Repeated integration by parts - similar as in the first case - implies

$$
|T| \leq c_{N} \cdot 2^{3 j / 2} \cdot\left\langle\left|b-\frac{m}{2 s_{j}}\right|_{D_{2^{j}}}\right\rangle^{-N}
$$

Claim (iii) now follows by reinserting $\theta$ and using (18).

3.2. Cluster of Curvelets. We can now prove negligible cluster coherence of $\mathcal{S}_{1, j}$.

Lemma 3.2.

$$
\left(\mu_{c}\right)_{1, j}=\mu_{c}\left(\mathcal{S}_{1, j},\left\{\left(g_{s_{j}}\right)_{\lambda}\right\} ;\left\{\gamma_{\eta}\right\}\right) \rightarrow 0, \quad \text { as } j \rightarrow \infty
$$

Proof. First, we observe that the definition of $\mathcal{S}_{1, j}$ implies

$$
\begin{aligned}
\left(\mu_{c}\right)_{1, j} & =\max _{m, n} \sum_{(b, \theta) \in \mathcal{S}_{1, j}}\left|\left\langle\gamma_{a_{j}, b, \theta},\left(g_{s_{j}}\right)_{m, n}\right\rangle\right| \\
& =\max _{m, n} \sum_{Q} \sum_{(b, \theta) \in \mathcal{S}_{1, j, Q}}\left|\left\langle\gamma_{a_{j}, b, \theta},\left(g_{s_{j}}\right)_{m, n}\right\rangle\right| \\
& \leq \# Q \cdot \max _{Q} \max _{m, n} \sum_{(b, \theta) \in \mathcal{S}_{1, j, Q}}\left|\left\langle\gamma_{a_{j}, b, \theta},\left(g_{s_{j}}\right)_{m, n}\right\rangle\right|
\end{aligned}
$$


Let us now first prove the claim in case that $\phi_{Q}=I d$, i.e., $M_{Q}=I d$, hence $\mathcal{S}_{1, j, Q}=\tilde{\mathcal{S}}_{j}$. Notice that WLOG we can assume that $m=0$ and $n=\left(2^{j}, 0\right)\left(\in M_{a_{j}, \theta, s_{j}}\right)$. Hence it remains to continue the estimate

$$
\left(\mu_{c}\right)_{1, j} \leq \# Q \cdot \max _{Q} \sum_{(b, \theta) \in \mathcal{S}_{1, j, Q}}\left|\left\langle\gamma_{a_{j}, b, \theta},\left(g_{s_{j}}\right)_{\left.0,2^{j}, 0\right)}\right\rangle\right| .
$$

We now split the proof - similar to the splitting in Lemma 3.1 - in three cases.

Case $s_{j}=o\left(2^{j / 2}\right)$ as $j \rightarrow \infty$. By (19) and Lemma 3.1,

$$
\begin{aligned}
\left(\mu_{c}\right)_{1, j} & \leq c_{N} \cdot 2^{-3 j / 4} \cdot s_{j} \sum_{(k, \ell) \in \tilde{\mathcal{S}}_{j}}\left\langle\left|s_{j}\left(2^{-j} k_{1}, 2^{-j / 2} k_{2}\right)\right|\right\rangle^{-N} \\
& \leq c_{N} \cdot 2^{-3 j / 4} \cdot s_{j} \cdot \pi^{-1} \cdot 2^{2 \varepsilon j} \sum_{\left|k_{2}\right| \leq 2 \rho+2^{-j(1 / 2-\varepsilon)}}\left\langle\left|s_{j} 2^{-j / 2} k_{2}\right|\right\rangle^{-N} \\
& \leq c_{N} \cdot 2^{-3 j / 4} \cdot s_{j} \cdot 2^{2 \varepsilon j} \cdot s_{j}^{-1} \cdot\left(2 \rho+2^{-j(1 / 2-\varepsilon)}\right) \\
& \leq c_{N} \cdot 2^{j\left(2 \varepsilon-\frac{3}{4}\right)} .
\end{aligned}
$$

Thus, since $\varepsilon<\frac{1}{8}$,

$$
\left(\mu_{c}\right)_{1, j} \rightarrow 0, \quad \text { as } j \rightarrow \infty
$$

which settles this case.

Case $s_{j}=\Omega\left(2^{j / 2}\right)$ and $s_{j}=o\left(2^{j}\right)$ as $j \rightarrow \infty$. By (19) and Lemma 3.1,

$$
\begin{aligned}
\left(\mu_{c}\right)_{1, j} & \leq c_{N} \cdot 2^{-j / 4} \sum_{(k, \ell) \in \tilde{\mathcal{S}}_{j}}\left\langle\left|\left(s_{j} b_{1}, 2^{j / 2} b_{2}\right)\right|_{R_{\theta}}\right\rangle^{-N} \\
& \leq c_{N} \cdot 2^{-j / 4} \cdot \pi^{-1} \cdot \sum_{\left|k_{1}\right| \leq 2^{\varepsilon j}} \sum_{\left|k_{2}\right| \leq 2 \rho+2^{-j(1 / 2-\varepsilon)}}\left\langle\left|\left(s_{j} 2^{-j} k_{1}, k_{2}\right)\right|\right\rangle^{-N} \\
& \leq c_{N} \cdot 2^{-j / 4} \cdot 2^{2 \varepsilon j} \sum_{\left|k_{2}\right| \leq 2 \rho+2^{-j(1 / 2-\varepsilon)}}\left\langle\left|k_{2}\right|\right\rangle^{-N}
\end{aligned}
$$

Since the last sum is bounded by a constant,

$$
\left(\mu_{c}\right)_{1, j} \leq c_{N} \cdot 2^{j\left(2 \varepsilon-\frac{1}{4}\right)} .
$$

Since $\varepsilon<\frac{1}{8}$

$$
\left(\mu_{c}\right)_{1, j} \rightarrow 0, \quad \text { as } j \rightarrow \infty
$$

which proves the claim for this case.

Case $s_{j}=\Omega\left(2^{j}\right)$ as $j \rightarrow \infty$. By (19) and Lemma 3.1,

$$
\begin{aligned}
\left(\mu_{c}\right)_{1, j} & \leq c_{N} \cdot 2^{3 j / 4} \cdot s_{j}^{-1} \sum_{(k, \ell) \in \tilde{\mathcal{S}}_{j}}\left\langle|b|_{D_{2^{j}} R_{\theta}}\right\rangle^{-N} \\
& \leq c_{N} \cdot 2^{3 j / 4} \cdot s_{j}^{-1} \cdot \pi^{-1} \cdot \sum_{k}\langle|k|\rangle^{-N} \\
& \leq c_{N} \cdot 2^{3 j / 4} \cdot s_{j}^{-1} .
\end{aligned}
$$


Hence,

$$
\left(\mu_{c}\right)_{1, j} \rightarrow 0, \quad \text { as } j \rightarrow \infty
$$

Hence also this case is proved.

The general claim now follows by using similar arguments as in [19, Lem. 8.8].

3.3. Cluster of Gabor Elements. Finally, we arrive at the study of the cluster coherence of $\mathcal{S}_{2, j}$.

Lemma 3.3. Suppose that one of conditions (i) and (ii) is satisfied:

(i) Suppose $s_{j}=o\left(2^{j}\right)$ as $j \rightarrow \infty$, and $r_{2, j}$ is chosen such that

$$
\left|B\left(0, r_{2, j}\right) \cap M_{a_{j}, 0, s_{j}} \cap \mathbb{Z}^{2}\right|=o\left(2^{3 j / 4} \cdot s_{j}^{-1}\right), \quad \text { as } j \rightarrow \infty .
$$

(ii) Suppose $s_{j}=\Omega\left(2^{j}\right)$ as $j \rightarrow \infty$, and $r_{1, j}$ is chosen such that

$$
\left|B\left(0, r_{1, j}\right) \cap \mathbb{Z}^{2}\right|=o\left(2^{-3 j / 4} \cdot s_{j}\right), \quad \text { as } j \rightarrow \infty .
$$

Then

$$
\left(\mu_{c}\right)_{2, j}=\mu_{c}\left(\mathcal{S}_{2, j},\left\{\gamma_{\eta}\right\} ;\left\{\left(g_{s_{j}}\right)_{\lambda}\right\}\right) \rightarrow 0, \quad \text { as } j \rightarrow \infty
$$

Proof. First,

$$
\begin{aligned}
\left(\mu_{c}\right)_{2, j} & =\max _{b, \theta} \sum_{(m, n) \in \mathcal{S}_{2, j}}\left|\left\langle\gamma_{a_{j}, b, \theta},\left(g_{s_{j}}\right)_{m, n}\right\rangle\right| \\
& =\sum_{(m, n) \in \mathcal{S}_{2, j}}\left|\left\langle\gamma_{a_{j}, 0,0},\left(g_{s_{j}}\right)_{m, n}\right\rangle\right|
\end{aligned}
$$

since, for symmetry reasons, WLOG we can assume that the maximum is attained in $b=0$ and $\theta=0$. The two cases will now be dealt with separately. We start with the first, which - similar to the splitting in Lemma 3.1 - we separate into two subcases.

Case $s_{j}=o\left(2^{j / 2}\right)$ as $j \rightarrow \infty$. By (20) and Lemma 3.1,

$$
\left(\mu_{c}\right)_{2, j} \leq c_{N} \cdot 2^{-3 j / 4} \cdot s_{j} \sum_{m \in B\left(0, r_{1, j}\right)}\left\langle\left|\frac{m}{2}\right|\right\rangle^{-N} \sum_{n \in B\left(0, r_{2, j}\right) \cap M_{a_{j}, 0, s_{j}} \cap \mathbb{Z}^{2}} 1 .
$$

Since the first sum is bounded by a constant and by condition (i),

$$
\left(\mu_{c}\right)_{2, j}=o(1), \quad \text { as } j \rightarrow \infty,
$$

this case is settled.

Case $s_{j}=\Omega\left(2^{j / 2}\right)$ and $s_{j}=o\left(2^{j}\right)$ as $j \rightarrow \infty$. By (20) and Lemma 3.1,

$$
\left(\mu_{c}\right)_{2, j} \leq c_{N} \cdot 2^{-j / 4} \sum_{m \in B\left(0, r_{1, j}\right)}\langle|\left(\frac{m_{1}}{2}, 2^{j / 2} \frac{m_{2}}{2 s_{j}}|\rangle^{-N} \sum_{n \in B\left(0, r_{2, j}\right) \cap M_{a_{j}, 0, s_{j}} \cap \mathbb{Z}^{2}} 1 .\right.
$$

Since

$$
\int_{0}^{r_{1, j}}\left\langle\left|2^{j / 2} s_{j}^{-1} x\right|\right\rangle^{-N} d x=2^{-j / 2} \cdot s_{j} \int_{0}^{2^{j / 2} s_{j}^{-1} r_{1, j}}\langle|x|\rangle^{-N} d x \sim 2^{-j / 2} \cdot s_{j},
$$


we obtain - also exploiting condition (i),

$$
\left(\mu_{c}\right)_{2, j} \leq c_{N} \cdot 2^{-3 j / 4} \cdot s_{j} \sum_{n \in B\left(0, r_{2, j}\right) \cap M_{a_{j}, 0, s_{j}} \cap \mathbb{Z}^{2}} 1=o(1), \quad \text { as } j \rightarrow \infty .
$$

This proves the claim for this case.

Case $s_{j}=\Omega\left(2^{j}\right)$ as $j \rightarrow \infty$. By (20) and Lemma 3.1,

$$
\left(\mu_{c}\right)_{2, j} \leq c_{N} \cdot 2^{3 j / 4} \cdot s_{j}^{-1} \sum_{m \in B\left(0, r_{1, j}\right)}\left\langle\left|b-\frac{m}{2 s_{j}}\right|_{D_{2 j}}\right\rangle^{-N} \sum_{n \in B\left(0, r_{2, j}\right) \cap M_{a_{j}, 0, s_{j}} \cap \mathbb{Z}^{2}} 1 .
$$

Since $\left|M_{a_{j}, 0, s_{j}} \cap \mathbb{Z}^{2}\right| \leq$ const,

$$
\begin{aligned}
\left(\mu_{c}\right)_{2, j} & \leq c_{N} \cdot 2^{3 j / 4} \cdot s_{j}^{-1} \sum_{m \in B\left(0, r_{1, j}\right)}\left\langle\left|\frac{1}{2}\left(2^{j} s_{j}^{-1} m_{1}, 2^{j / 2} s_{j}^{-1} m_{2}\right)\right|\right\rangle^{-N} \\
& \leq c_{N} \cdot 2^{3 j / 4} \cdot s_{j}^{-1}\left|\left\{m \in \mathbb{Z}^{2}:|m| \leq r_{1, j}\right\}\right| .
\end{aligned}
$$

By condition (ii),

$$
\left(\mu_{c}\right)_{2, j}=o(1), \quad \text { as } j \rightarrow \infty,
$$

The lemma is proved.

\section{Proofs}

\subsection{Proofs of Results from Section 1.}

4.1.1. Proof of Lemma 1.1. Proof. We first observe that, for $W$ sufficiently nice,

$$
\left\|\mathcal{C}_{j}\right\|_{2}^{2}=\int\left|W\left(a_{j}|\xi|\right)\right|^{2}\left|\hat{\mathcal{C}}_{j}(\xi)\right|^{2} d \xi \sim \int_{\tilde{\mathcal{A}}_{j}}|\hat{\mathcal{C}}(\xi)|^{2} d \xi
$$

where $\tilde{\mathcal{A}}_{j}=\left\{\xi: 2^{j} \leq|\xi|<2^{j+1}\right\}$. Using (3), we can continue (21) to

$$
\left\|\mathcal{C}_{j}\right\|_{2}^{2} \sim \int_{2^{j}}^{2^{j+1}} r^{-2} d r \sim 2^{-j}
$$

4.1.2. Proof of Lemma 1.2. First, by the change of variable $\omega=\xi / s$ and the support condition on $\hat{g}$,

$$
\begin{aligned}
\left\|\mathcal{T}_{s, j}\right\|_{2}^{2} & =\sum_{m, n} \sum_{\tilde{m}, \tilde{n}} \int d_{m, n} \overline{d_{\tilde{m}, \tilde{n}}} W^{2}\left(a_{j}|\xi|\right) \hat{g}_{s}(\xi-s n) \overline{\hat{g}_{s}(\xi-s \tilde{n})} e^{i \frac{m-\tilde{m}}{2 s} \xi} d \xi \\
& =s^{2} \cdot \sum_{\substack{m, n, \tilde{m}, \tilde{n} \\
|n-\tilde{n}| \leq 1}} \int d_{m, n} \overline{d_{\tilde{m}, \tilde{n}}} W^{2}\left(a_{j} s|\omega|\right) \hat{g}_{s}(s(\omega-n)) \overline{\hat{g}_{s}(s(\omega-\tilde{n}))} e^{i \frac{m-\tilde{m}}{2} \omega} d \omega \\
& =\sum_{\substack{m, n, \tilde{m}, \tilde{n} \\
|n-\tilde{n}| \leq 1}} d_{m, n} \overline{d_{\tilde{m}, \tilde{n}}} \int W^{2}\left(a_{j} s|\omega|\right) \hat{g}(\omega-n) \overline{\hat{g}(\omega-\tilde{n})} e^{i \frac{m-\tilde{m}}{2} \omega} d \omega .
\end{aligned}
$$


For 'sufficiently nice' $W$,

$$
\int W^{2}\left(a_{j} s|\omega|\right) \hat{g}(\omega-n) \overline{\hat{g}(\omega-\tilde{n})} e^{i \frac{m-\tilde{m}}{2} \omega} d \omega \sim \int_{\mathcal{A}_{s, j}} \hat{g}(\omega-n) \overline{\hat{g}(\omega-\tilde{n})} e^{i \frac{m-\tilde{m}}{2} \omega} d \omega
$$

hence, continuing (22), and taking into account that for each $n$, there exist a finite number of $\tilde{n}$ 's independent on $j$ satisfying $|n-\tilde{n}| \leq 1$,

$$
\begin{aligned}
\left\|\mathcal{T}_{s, j}\right\|_{2}^{2} & \sim \sum_{\substack{m, n, \tilde{m}, \tilde{n} \\
|n-\tilde{n}| \leq 1}} d_{m, n} \overline{d_{\tilde{m}, \tilde{n}}} \int_{\mathcal{A}_{s, j}} \hat{g}(\omega-n) \overline{\hat{g}(\omega-\tilde{n})} e^{i \frac{m-\tilde{m}}{2} \omega} d \omega \\
& =\sum_{\substack { m, \tilde{m} \\
\begin{subarray}{c}{n, \tilde{n} \in \mathbb{Z}^{2} \cap \mathcal{A}_{s, j} \\
|n-\tilde{n}| \leq 1{ m , \tilde { m } \\
\begin{subarray} { c } { n , \tilde { n } \in \mathbb { Z } ^ { 2 } \cap \mathcal { A } _ { s , j } \\
| n - \tilde { n } | \leq 1 } }\end{subarray}} d_{m, n} \overline{d_{\tilde{m}, \tilde{n}}} \int_{\mathcal{A}_{s, j}} \hat{g}(\omega-n) \overline{\hat{g}(\omega-\tilde{n})} e^{i \frac{m-\tilde{m}}{2} \omega} d \omega \\
& \sim \sum_{m, \tilde{m}} \sum_{n \in \mathbb{Z}^{2} \cap \mathcal{A}_{s, j}} d_{m, n} \overline{d_{\tilde{m}, n}} \int_{\mathcal{A}_{s, j}}|\hat{g}(\omega-n)|^{2} e^{i \frac{m-\tilde{m}}{2} \omega} d \omega .
\end{aligned}
$$

Now, WLOG we assume that the support of $\hat{g}$ is always entirely contained in $\mathcal{A}_{s, j}$; then, by $(23)$

$$
\left\|\mathcal{T}_{s, j}\right\|_{2}^{2} \sim \sum_{m, \tilde{m}} \sum_{n \in \mathbb{Z}^{2} \cap \mathcal{A}_{s, j}} d_{m, n} \overline{d_{\tilde{m}, n}} \int_{\mathbb{R}^{2}}|\hat{g}(\omega-n)|^{2} e^{i \frac{m-\tilde{m}}{2} \omega} d \omega
$$

Since $|g(x)| \sim e^{-|x|}$,

$$
\begin{aligned}
\int_{\mathbb{R}^{2}}|\hat{g}(\omega-n)|^{2} e^{i \frac{m-\tilde{m}}{2} \omega} d \omega & =e^{i \frac{m-\tilde{m}}{2} n} \cdot \int_{\mathbb{R}^{2}}|\hat{g}(\omega)|^{2} e^{i \frac{m-\tilde{m}}{2} \omega} d \omega \\
& =e^{i \frac{m-\tilde{m}}{2} n} \cdot\left(g \star g^{\star}\right)\left(\frac{m-\tilde{m}}{2}\right) \\
& \sim e^{-\frac{|m-\tilde{m}|}{2}} .
\end{aligned}
$$

Thus, by (24),

$$
\left\|\mathcal{T}_{j}\right\|_{2}^{2} \sim \sum_{m, \tilde{m}} \sum_{n \in \mathbb{Z}^{2} \cap \mathcal{A}_{s, j}} d_{m, n} \overline{d_{\tilde{m}, n}} e^{-\frac{|m-\tilde{m}|}{2}}
$$

and the lemma is proved.

4.1.3. Proof of Theorem 1.1. The theorem follows immediately from applying Lemmata 2.4, 2.3, 3.3, and 3.2 to Proposition 1.1.

4.1.4. Proof of Corollary 1.1. We choose $r_{1, j}>0$ such that

$$
r_{1, j}=\omega\left(2^{\frac{1}{2 \delta(1+\delta)} j}\right), \quad \text { as } j \rightarrow \infty,
$$

and $r_{2, j}>0$ such that

$$
r_{2, j}=\omega\left(2^{\frac{1}{2(1+\delta)} j}\right), \quad \text { as } j \rightarrow \infty
$$


It now suffices to check the sufficient conditions posed in Theorem 1.1. Firstly, by Example 2.1 ,

$$
\sum_{\substack{(m, n) \notin B\left(0, r_{1, j}\right) \times B\left(0, r_{2, j}\right) \\ n \in \mathcal{A}_{j}}} \sum_{\tilde{m} \in \mathbb{Z}^{2}}\left|d_{\tilde{m}, n}\right| e^{-\frac{|\tilde{m}-m|}{2}}=o\left(2^{-j / 2}\right), \quad j \rightarrow \infty .
$$

Now notice that the energy matching condition in this case, i.e., (7), implies that $s_{j}=\Omega\left(2^{j / 2}\right)$ and $s_{j}=o\left(2^{j}\right)$ as $j \rightarrow \infty$. Hence we need to prove

$$
\left|B\left(0, r_{2, j}\right) \cap M_{a_{j}, 0, s_{j}} \cap \mathbb{Z}^{2}\right|=o\left(2^{3 j / 4} \cdot s_{j}^{-1}\right)=o\left(2^{\frac{1-\delta}{4(1+\delta)} j}\right), \quad \text { as } j \rightarrow \infty .
$$

For this, by (25),

$$
\left|B\left(0, r_{2, j}\right) \cap M_{a_{j}, 0, s_{j}} \cap \mathbb{Z}^{2}\right|=\left|M_{a_{j}, 0, s_{j}} \cap \mathbb{Z}^{2}\right| \sim s_{j}^{-1} \cdot 2^{j} \cdot s_{j}^{-1} \cdot 2^{j / 2}=s_{j}^{-2} \cdot 2^{3 j / 2}, \quad \text { as } j \rightarrow \infty .
$$

Since, by (7),

$$
s_{j}^{-2} \cdot 2^{3 j / 2}=2^{\frac{1-\delta}{2(1+\delta)} j},
$$

claim (26) is satisfied if and only if $\delta>1$, which was assumed.

The claim now follows from Theorem 1.1.

\section{REFERENCES}

[1] J.-F. Aujol, G. Aubert, L. Blanc-Féraud, and A. Chambolle, Image decomposition into a bounded variation component and an oscillating component, J. Math. Imaging Vision 22(1) (2005), 71-88.

[2] O. Ben-Shahar and S. W. Zucker, The Perceptual Organization of Texture Flow: A Contextual Inference Approach, IEEE Trans. Pattern Anal. 25(4) (2003), 401-417.

[3] M. Bertalmyo, L. Vese, G. Sapiro, and S. Osher, Simultaneous structure and texture image inpainting, IEEE Trans. Image Process. 12(8) (2003), 882-889.

[4] J. Bobin, J.-L. Starck, M.J. Fadili, Y. Moudden, and D.L. Donoho, Morphological Component Analysis: An Adaptive Thresholding Strategy, IEEE Trans. Image Process. 16(11) (2007), 2675-2681.

[5] E. J. Candès and D. L. Donoho, New tight frames of curvelets and optimal representations of objects with piecewise $C^{2}$ singularities, Comm. Pure Appl. Math. 56(2) (2004), 219-266.

[6] E. J. Candès and D. L. Donoho, Continuous curvelet transform: I. Resolution of the wavefront set, Appl. Comput. Harmon. Anal. 19(2) (2005), 162-197.

[7] E. J. Candès and D. L. Donoho, Continuous curvelet transform: II. Discretization of frames, Appl. Comput. Harmon. Anal. 19(2) (2005), 198-222.

[8] E. J. Candès, J. K. Romberg, and T. Tao, Stable signal recovery from incomplete and inaccurate measurements, Comm. Pure Appl. Math. 59(8) (2006), 1207-1223.

[9] S. S. Chen, D. L. Donoho, and M. A. Saunders, Atomic decomposition by basis pursuit, SIAM Rev. 43 (2001), 129-159.

[10] M. Clerc and S. Mallat, The texture gradient equation for recovering shape from texture, IEEE Trans. Pattern Anal. 24(4) (2002), 536-549.

[11] R. R. Coifman and M. V. Wickerhauser, Wavelets and adapted waveform analysis. A toolkit for signal processing and numerical analysis, Different perspectives on wavelets (San Antonio, TX, 1993), 119-153, Proc. Sympos. Appl. Math., 47, Amer. Math. Soc., Providence, RI, 1993.

[12] I. Daubechies, A. Grossman, and Y. Meyer, Painless nonorthogonal expansions, Journal Math. Phys. 27 (1986), 1271-1283.

[13] D. L. Donoho, Compressed sensing, IEEE Trans. Inform. Theory 52(4) (2006), 1289-1306.

[14] D. L. Donoho, For most large underdetermined systems of linear equations the minimal $l_{1}$-norm solution is also the sparsest solution, Comm. Pure Appl. Math. 59(6) (2006), 797-829. 
[15] D. L. Donoho, For most large underdetermined systems of equations, the minimal $l_{1}$-norm near-solution approximates the sparsest near-solution, Comm. Pure Appl. Math. 59(7) (2006), 907-934.

[16] D. L. Donoho and M. Elad, Optimally sparse representation in general (nonorthogonal) dictionaries via $l^{1}$ minimization, Proc. Natl. Acad. Sci. USA 100(5) (2003), 2197-2202.

[17] D. L. Donoho, M. Elad, and V. N. Temlyakov, Stable recovery of sparse overcomplete representations in the presence of noise, IEEE Trans. Inform. Theory 52(1) (2006), 6-18.

[18] D. L. Donoho and X. Huo, Uncertainty principles and ideal atomic decomposition, IEEE Trans. Inform. Theory 47(7) (2001), 2845-2862.

[19] D. L. Donoho and G. Kutyniok, Microlocal Analysis of the Geometric Separation Problem, Comm. Pure Appl. Math., to appear.

[20] D. L. Donoho and B. F. Logan, Signal recovery and the large sieve, SIAM J. Appl. Math. 52(2) (1992), $577-591$.

[21] D. L. Donoho and P. B. Stark, Uncertainty principles and signal recovery, SIAM J. Appl. Math. 49(3) (1989), 906-931.

[22] B. Dubuc and S. W. Zucker, Complexity, Confusion, and Perceptual Grouping. Part I: the curve like representation, Int. J. of Computer Vision 42(1/2) (2001), 55-82, 2001.

[23] B. Dubuc and S. W. Zucker, Complexity, Confusion, and Perceptual Grouping. Part II: mapping complexity, Int. J. of Computer Vision, 42(1/2) (2001), 83-115, 2001.

[24] M. Elad and A. M. Bruckstein, A Generalized Uncertainty Principle and Sparse Representation in Pairs of Bases, IEEE Trans. Inform. Theory 48(9) (2002), 2558-2567.

[25] M. Elad, J.-L. Starck, P. Querre, and D. L. Donoho, Simultaneous cartoon and texture image inpainting using morphological component analysis (MCA), Appl. Comput. Harmon. Anal. 19(3) (2005), 340-358.

[26] S. Esedoglu and S. J. Osher, Decomposition of images by the anisotropic Rudin-Osher-Fatemi model, Comm. Pure Appl. Math. 57(12) (2004), 1609-1626.

[27] J. B. Garnett, T. M. Le, Y. Meyer, and L. A. Vese, Image decompositions using bounded variation and generalized homogeneous Besov spaces, Appl. Comput. Harmon. Anal. 23(1) (2007), 25-56.

[28] J.-B. Gouéré, Quasicrystals and almost periodicity, Commun. Math. Phys. 255 (2005), 655-681.

[29] R. Gribonval and E. Bacry, Harmonic decomposition of audio signals with matching pursuit, IEEE Trans. Signal Process. 51(1) (2003), 101-111.

[30] R. Gribonval and M. Nielsen, Sparse representations in unions of bases, IEEE Trans. Inform. Theory 49(12) (2003), 3320-3325.

[31] K. Guo, G. Kutyniok, and D. Labate, Sparse Multidimensional Representations using Anisotropic Dilation und Shear Operators, in Wavelets und Splines (Athens, GA, 2005), G. Chen und M. J. Lai, eds., Nashboro Press, Nashville, TN (2006), 189-201.

[32] K. Guo and D. Labate, Optimally sparse multidimensional representation using shearlets, SIAM J. Math. Anal. 39 (2007), 298-318.

[33] A. Haddad, Texture separation $B V-G$ and $B V-L^{1}$ models, Multiscale Model. Simul. 6(1) (2007), 273-286.

[34] A. Haddad and Y. Meyer, An improvement of Rudin-Osher-Fatemi model, Appl. Comput. Harmon. Anal. 22(3) (2007), 319-334.

[35] E. Hlawka, Über Integrale auf konvexen Körpern. I, Monatsh. Math. 54 (1950), 1-36.

[36] L. Hörmander, The analysis of linear partial differential operators. I. Distribution theory and Fourier analysis. Springer-Verlag, Berlin, 2003.

[37] P. Kittipoom, G. Kutyniok, and W.-Q Lim, Construction of Compactly Supported Shearlet Frames, Constr. Approx. 35(1) (2012), 21-72.

[38] G. Kutyniok, Data Separation by Sparse Representations, in: Compressed Sensing: Theory and Applications, Cambridge University Press, 2012.

[39] G. Kutyniok, Geometric Separation by Single-Pass Alternating Thresholding, preprint.

[40] G. Kutyniok, Sparsity Equivalence of Anisotropic Decompositions, preprint.

[41] G. Kutyniok and W.-Q Lim, Compactly Supported Shearlets are Optimally Sparse, J. Approx. Theory 163(11) (2011), 1564-1589. 
[42] G. Kutyniok and D. Labate, Resolution of the Wavefront Set using Continuous Shearlets, Trans. Amer. Math. Soc. 361 (2009), 2719-2754.

[43] T. M. Le and L. A. Vese, Image decomposition using total variation and $\operatorname{div}(\mathrm{BMO})$, Multiscale Model. Simul. 4(2) (2005), 390-423.

[44] F. G. Meyer, A. Averbuch, and R. R. Coifman, Multi-layered Image Representation: Application to Image Compression, IEEE Trans. Image Process. 11(9) (2002), 1072-1080.

[45] Y. Meyer, Oscillating Patterns in Image Processing and in Some Nonlinear Evolution Equations, The Fifteenth Dean Jacqueline B. Lewis Memorial Lectures, Univ. Lecture Ser. 22, AMS, Providence, RI, 2001.

[46] B. A. Olshausen and D. J. Field, Emergence of Simple-Cell Receptive Field Properties by Learning a Sparse Code for Natural Images, Nature 381 (1996), 607-609.

[47] S. Osher, A. Solé, and L. Vese, Image decomposition and restoration using total variation minimization and the $H^{-1}$ norm, Multiscale Model. Simul. 1(3) (2003), 349-370.

[48] B. Randol, On the Fourier transform of the indicator function of a planar set, Trans. Amer. Math. Soc. 139 (1969), 271-278.

[49] J.-L. Starck, M. Elad, and D. L. Donoho, Image Decomposition: Separation of Texture from Piece-Wise Smooth Content, Wavelets X (San Diego, CA, 2003), SPIE Proc. 5207, SPIE, Bellingham, WA, 2003.

[50] J.-L. Starck, M. Elad, and D. L. Donoho, Redundant Multiscale Transforms and their Application for Morphological Component Analysis, Journal of Advances in Imaging and Electron Physics 132 (2004), 287-348.

[51] J.-L. Starck, M. Elad, and D. L. Donoho, Image decomposition via the combination of sparse representations and a variational approach, IEEE Trans. Image Proc. 14(10) (2005), 1570-1582.

[52] J.-L. Starck, Y. Moudden, J. Bobin, M. Elad, and D.L. Donoho, Morphological Component Analysis, Wavelets XI (San Diego, CA, 2005), SPIE Proc. 5914, SPIE, Bellingham, WA, 2005.

[53] J.-L. Starck, M. Nguyen, and F. Murtagh, Wavelets and curvelets for image deconvolution: A combined approach, Signal Process. 83 (2003), 2279-2283.

[54] E. Tadmor, S. Nezzar, and L. Vese, A multiscale image representation using hierarchical $\left(B V, L^{2}\right)$ decompositions, Multiscale Model. Simul. 2(4) (2004), 554-579.

[55] J. A. Tropp, Greed is good: algorithmic results for sparse approximation, IEEE Trans. Inform. Theory 50(10) (2004), 2231-2242.

[56] L. A. Vese and S. J. Osher, Modeling Textures with Total Variation Minimization and Oscillating Patterns in Image Processing, J. Sci. Comput. 19(1-3) (2003), 553-572.

[57] S.-C. Zhu, C. Guo, Y. Wang, and Z. Xu, What are Textons?, Int. J. Comput. Vision 62(1-2) (2005), 121-143.

[58] M. Zibulevsky and B. Pearlmutter, Blind source separation by sparse decomposition in a signal dictionary, Neur. Comput. 13 (2001), 863-882.

Department of Mathematics, Technische Universität Berlin, 10623 Berlin, Germany E-mail address: kutyniok@math.tu-berlin.de 